\title{
La FMH et les Médecins en faveur de l'Environnement (MfE) réclament des prescriptions plus sévères
}

\section{Bernhard Aufdereggen, \\ Yvonne Gilli, \\ Cornelia Semadeni, \\ Edith Steiner}

Groupe de travail «Champs électromagnétiques» des MfE

1 Ivancsits S, Diem E, Jahn O, Rüdiger H.W. Intermittent extremely low frequency electromagnetic fields cause DNA damage in a dosedependent way. Int. Arch. Occup. Environ. Health 2003b;76:431-436.

2 Ruediger HW. Genotoxic effects of radiofrequency electromagnetic fields, Pathophysiology. 2009. doi:10.1016/j.pathophys.200 8.11.004.

3 Hug K, Rapp R, Schär P, Taschner N. Hochfrequente Strahlung und Gesundheit. Bewertung von wissenschaftlichen Studien im Niedrigdosisbereich. Umwelt-Wissen. 2006. Nr. 0722. Bundesamt für Umwelt, Bern.

4 IARC Working Group on the Evaluation of Carcinogenic Risks to Humans. Non-ionizing radiation, Part 1: static and extremely lowfrequency (ELF) electric and magnetic fields. IARC Monogr Eval Carcinog Risks Hum. 2002;80:1-395.

\begin{abstract}
Introduction
L'actuelle ordonnance RNI a été édictée le 23 décembre 1999. Depuis lors, le niveau d'exposition de la population suisse au rayonnement non ionisant (RNI) a considérablement augmenté. Les données scientifiques concernant des atteintes à la santé à des doses inférieures aux valeurs limites légales se multiplient. On soupçonne les champs magnétiques d'être cancérigènes. Les personnes habitant à proximité de lignes à haute tension encourent un plus grand risque d'être atteintes d'Alzheimer. Des indices suggèrent une corrélation entre un risque accru de tumeur cérébrale et l'usage de longue durée de téléphones portables. En laboratoire, des modifications du patrimoine génétique de certains types de cellules ont été mises en évidence lors d'exposition à un RNI. Cinq pour cent de la population est électrosensible. D'un point de vue médical, le principe de précaution doit s'appliquer dès à présent en ce qui concerne l'exposition au RNI. Or, le projet de révision de l'ORNI en cours de consultation ne satisfait pas à cette exigence.
\end{abstract}

\section{Bases légales}

L'ordonnance RNI régit la protection de la population contre le rayonnement non ionisant émis par des installations stationnaires, en tenant compte des dispositions de la loi sur la protection de l'environnement (LPE). Elle a été mise en vigueur par le Conseil fédéral le $1^{\text {er }}$ février 2000 . Les valeurs limites d'immission alors définies l'ont été sur la base des recommandations de la Commission internationale de protection contre le rayonnement non ionisant (ICNIRP). Elles protègent contre des effets nocifs étayés scientifiquement, par exemple l'échauffement des tissus dû à un fort rayonnement de la téléphonie mobile, ou encore l'excitation musculaire et nerveuse provoquée par de puissants champs magnétiques. Déjà connus à l'époque, les effets biologiques d'une exposition à de faibles doses, tout comme les effets potentiellement négatifs à long terme, n'ont en revanche pas été pris en considération. Pour néanmoins tenir compte du principe de précaution inscrit dans la LPE, l'exposition au RNI dans des lieux où des personnes séjournent de manière prolongée a été restreinte plus sévèrement par une limitation des émissions des installations en tant que telles (valeur limite de l'installation).
La fixation des valeurs limites de l'installation n'a pas été fondée sur des critères biologiques, mais sur des critères de faisabilité, à savoir qu'elle tient compte des conditions techniques d'exploitation et des critères d'économicité. De plus, de généreuses dérogations sont prévues en ce qui concerne l'exposition à des champs magnétiques. Les valeurs limites d'immission applicables aux champs magnétiques des lignes à haute tension sont fixées à $100 \mu \mathrm{T}$, celles applicables au rayonnement de la téléphonie mobile à 40-60 V/m; les valeurs limites des installations s'élèvent à $1 \mu \mathrm{T}$ respectivement $0,4-0,6 \mathrm{~V} / \mathrm{m}$. Aucune prescription légale n'a été édictée pour les appareils mobiles tels que les téléphones portables. Il existe simplement des valeurs indicatives internationales, mais qui ne tiennent pas compte de la primauté du principe de précaution inscrit dans la LPE suisse.

\section{Doses inférieures aux valeurs limites légales peut-être dangereuses}

Dix ans après la mise en consultation de l'ORNI, les données scientifiques sur les effets biologiques du RNI à des doses situées en-dessous des valeurs limites d'immission sont toujours insuffisantes, tout particulièrement en ce qui concerne les effets à long terme et les groupes de population sensibles comme les enfants, les femmes enceintes et les personnes malades. La question des effets à long terme requiert des études étalées sur plusieurs années. Les épidémiologistes se heurtent à des difficultés pour évaluer l'exposition, ainsi qu'à des problèmes méthodologiques. Les résultats des travaux de recherche sont contradictoires. Nos connaissances demeurent très lacunaires. Il existe très peu d'études consacrées au rayonnement des stations de base de la téléphonie mobile. Les effets sur les enfants n'ont pratiquement pas encore été étudiés. Les considérations relatives aux modes d'action du RNI restent hypothétiques. La recherche et la protection de la santé sont à la traîne du progrès technique. Par exemple, encore aucune étude n'a été réalisée quant aux effets sur la santé des liaisons informatiques sans fil (WLAN), alors que leur introduction à grande échelle est en cours. L'imbrication entre industrie et recherche se renforce. Il est prouvé que les résultats de nombreuses études scientifiques sont dépendants de l'industrie. 
5 Garcia AM, Sisternas A, Hoyos SP. Occupational exposure to extremely low frequency electric and magnetic fields and Alzheimer disease: a meta-analysis. Int J Epidemiol. 2008;37(2) 329-340.

6 Huss A, Spoerri A, Egger M, Röösli M. for the Swiss National Cohort Study. Residence Near Power Lines and Mortality From Neurodegenerative Diseases. Longitudinal Study of the Swiss Population. Am J Epidemiol. 2009;169(2): 167-175.

7 International Agency for Research on cancer. IARC World Cancer Report 2008. Section 2. Etiology of cancer. Chapter 2.12. Electromagnetic radiation: $170 \mathrm{ff}$.

8 Kundi M. The Controversy about a possible relationship between mobile phone use and cancer. A review. Environmental Health Perspectives. 2009;117(3)

9 Kundi M, Hutter H-P. Mobile phone base stations - Effects on wellbeing and health, Pathophysiology. 2009 doi:10.1016/j.pathophys.20 09.01.008.

10 Schreier et al. The Prevalence of Symptoms Attributed to Electromagnetic Field Exposure: A Cross Sectional Representative Survey in Switzerland. Soz Preventive Med. 2009;51:202-209.

11 Röösli M. Radiofrequency electromagnetic field exposure and non-specific symptoms of ill health. A systematic review. Environmental Research. 2008; 107(2):277-287.

12 Huss A, Küchenhoff J, Bircher A et al. Symptoms attributed to the environment. A systematic, interdisciplinary assessment. Int J Hyg Environ Health. 2004; 207(3):245-54.

13 www.qualifex.ch (Projekt des Nationalen Forschungsprogramms NFP 57 .Nichtionisierende Strahlung Umwelt und Gesundheit).
Par rapport à l'état des connaissances en 1999, des données scientifiques de plus en plus convergentes laissent penser que le RNI est nocif même en-dessous des valeurs limites en vigueur. En laboratoire, des modifications du patrimoine génétique $[1,2]$ et du métabolisme [3] de certains types de cellules ont été mises en évidence dans certaines conditions d'exposition à un RNI d'une intensité inférieure aux valeurs limites. Des études menées au sein de populations habitant à proximité de lignes à haute tension ont fait apparaître un risque de leucémie deux fois plus élevé chez les enfants en cas d'exposition permanente à un champ magnétique d'une intensité de 0,3-0,4 $\mu \mathrm{T}$. C'est ce qui a amené le Centre international de recherche sur le cancer (CIRC) à classer les champs magnétiques à basse fréquence comme «potentiellement cancérogènes pour l'homme» (2002) [4]. L'évidence épidémiologique d'un lien entre l'exposition à des champs magnétiques dans le cadre professionnel et le risque de maladies démentielles s'est renforcée ces dernières années [5]. Une étude bernoise, dont les résultats ont été publiés tout récemment, révèle un risque d'Alzheimer deux fois plus élevé chez les personnes séjournant de façon prolongée à proximité de lignes à haute tension [6]. La recherche sur le risque de tumeur cérébrale en cas d'usage de longue durée de téléphones portables suggère que celui-ci pourrait légèrement augmenter avec une durée d'utilisation de plus de 10 ans chez les gros utilisateurs $[7,8]$. Quelques-unes des rares études consacrées au rayonnement des stations de base de la téléphonie mobile mettent en évidence un accroissement de la fréquence de troubles du bien-être, principalement céphalées et difficultés de concentration, liés au degré d'exposition au rayonnement [9]. Cinq pour cent de la population suisse présente ou a déjà présenté des troubles attribués au RNI (28\% en lien avec des lignes à haute tension, 18\% en lien avec des stations de base) [10]. En laboratoire, il n'a pas été possible à ce jour d'objectiver le phénomène de l'électrosensibilité en cas d'exposition de courte durée, ni d'en prouver les effets immédiats [11]. Dans le cadre d'un projet pilote bâlois mené par un service-conseil en médecine environnementale, un lien de causalité avec le RNI a néanmoins pu être attesté par une équipe interdisciplinaire d'experts chez un tiers des patientes et patients électrosensibles examinés, quand bien même les valeurs limites en vigueur étaient respectées [12]

\section{Augmentation considérable de I'exposition quotidienne au RNI}

La téléphonie mobile s'est développée à un rythme inouï dans l'ensemble de la population en l'espace de quelques années, et le nombre d'abonnements de téléphonie mobile est passé de 3 millions en 1999 à 8 millions en 2007. D'énormes progrès ont été réalisés dans les technologies de transmission. La communication mobile et la transmission de données en tout temps et de tout lieu vers tout lieu au moyen d'équipements multimédia et multifonctions faciles d'usage n'est plus une vision d'avenir mais deviendra réalité dans un futur proche. En 2007, près de deux nouvelles antennes de téléphonie mobile ont été mises en service chaque jour. Le nombre d'antennes de téléphonie mobile est passé de tout juste 3000 en 1998, année de mise en consultation de l'ORNI, à plus de 12000 en 2007. On annonce déjà l'introduction de la quatrième génération de téléphonie mobile. La densité d'implantation des antennes continuera d'augmenter. En raison de la croissance démographique et économique, la consommation d'électricité a progressé de $24 \%$ entre 1990 et 2006. Il est prévu de construire ou transformer d'ici à 2015 un total de 67 lignes à haute tension.

Les premiers résultats de l'étude Qualifex [13] sur l'exposition de la population suisse au rayonnement issu de la communication mobile, montrent que les principales sources d'exposition sont les antennes de téléphonie mobile (32,02\%), les téléphones portables (29,10\%), la téléphonie sans fil DECT (22,75\%) et, pour une part encore faible, les liaisons informatiques sans fil $(4,14 \%)$.

\section{La prévention s'impose}

En 2000 déjà, la Fédération des médecins suisse (FMH) et les Médecins en faveur de l'Environnement (MfE) avaient demandé que, en lieu et place de la valeur limite de l'installation fixée par la loi à $6 \mathrm{~V} / \mathrm{m}$, une valeur limite d'immission pour les services de radiocommunication soit fixée à $0,6 \mathrm{~V} / \mathrm{m}$, à titre préventif, pour protéger la santé publique. A l'occasion de la dernière révision de l'ORNI, la FMH et les MfE ont réitéré leur demande d'abaissement des valeurs limites. De plus, les dérogations rendues possibles en cas de dépassement de la valeur limite de l'installation, doivent être supprimées. La fonction préventive de la valeur limite de l'installation ne doit pas être diluée, comme le laissent craindre les modifications de l'ordonnance proposées dans le projet mis en consultation.

L'augmentation énorme des niveaux d'exposition quotidienne au RNI exige une approche globale. Outre un renforcement des dispositions légales de protection, nous réclamons des efforts de recherche soutenus, la création d'un registre des cancers ainsi que le recensement systématique d'éventuels effets du RNI sur la santé des riveraines et riverains d'installations et sur celle des utilisatrices et utilisateurs d'appareils émettant un RNI. Il est urgent de propager une prévoyance à l'échelon individuel par une information large et spécifique de toute la population sur les mesures qui permettent de diminuer l'exposition quotidienne au RNI issu de sources domestiques. 